\title{
Numerical Model Describing the Early Age Behavior of 3D Printed Concrete - Work in Progress
}

\author{
Andersen, Sebastian; da Silva, Wilson Ricardo Leal; Paegle, leva; Nielsen, Jens Henrik
}

Published in:

Second RILEM International Conference on Concrete and Digital Fabrication

Link to article, DOI:

10.1007/978-3-030-49916-7_18

Publication date:

2020

Document Version

Peer reviewed version

Link back to DTU Orbit

Citation (APA):

Andersen, S., da Silva, W. R. L., Paegle, I., \& Nielsen, J. H. (2020). Numerical Model Describing the Early Age Behavior of 3D Printed Concrete - Work in Progress. In Second RILEM International Conference on Concrete and Digital Fabrication: Digital Concrete 2020 (Vol. 28, pp. 175-184). Springer. RILEM Bookseries Vol. 28 https://doi.org/10.1007/978-3-030-49916-7_18

\section{General rights}

Copyright and moral rights for the publications made accessible in the public portal are retained by the authors and/or other copyright owners and it is a condition of accessing publications that users recognise and abide by the legal requirements associated with these rights.

- Users may download and print one copy of any publication from the public portal for the purpose of private study or research.

- You may not further distribute the material or use it for any profit-making activity or commercial gain

- You may freely distribute the URL identifying the publication in the public portal 


\title{
Numerical Model Describing the Early Age Behavior of 3D Printed Concrete - work in progress
}

\author{
Sebastian Andersen ${ }^{1}$, Wilson Ricardo Leal da Silva ${ }^{2}$, Ieva Paegle ${ }^{1}$ and Jens Henrik \\ Nielsen ${ }^{1}$ \\ ${ }^{1}$ Technical University of Denmark, 2800 Kgs. Lyngby, Denmark \\ ${ }^{2}$ Danish Technological Institute, 2630 Taastrup, Denmark \\ seba@byg.dtu.dk
}

\begin{abstract}
A numerical model used to predict the behavior of 3D printed concrete in the early age phase up until the point of collapse is presented. An incremental creep model for the material including aging and temperature effects is suggested. Furthermore, kinematic nonlinear effects are included in order to predict instability. The input parameters to the numerical model are determined through an optimization process where the displacement error between a finite element method (FEM) model and digital image correlation (DIC) measurements from an actual 3D print are minimized. A simplified (current state) version of the suggested numerical model was used to predict at how many layers a specific 3D print collapsed, with an error of $58 \%$. This was concluded to be expected, since the numerical model is not fully implemented yet.
\end{abstract}

Keywords: 3D Concrete Print, Creep, Digital Image Correlation, Finite Element Method, Optimization loop.

\section{Introduction}

Additive manufacturing, also known as 3D printing, has experienced a rapid development over the past decades within the construction industry, see e.g. Buchanan and Gardner (2018). The principle of the method consist in using an automated process to construct a defined 3D model by sequentially adding layers on top of each other.

In the concrete industry the method is often referred to as $3 \mathrm{D}$ concrete printing (3DCP). However, one of the major challenges in 3DCP is the absence of formwork, which implies that the printed concrete should be able to carry it's own weight (and potential exterior loads) immediately upon printing. Otherwise collapse may occur. This requirement is typically referred to as buildability, cf. Buswell et al. (2018).

To prevent collapse during printing, knowledge about the concrete behavior in the early age stage is required. For this purpose, analytical and numerical models using input parameters from experimental tests can be used. Wolf et al. (2018) and Wolf et al. (2019) used ABAQUS with input parameters determined from triaxial compression tests and shear tests models to predict the mechanical behavior of 3DCPs in the early age stage. Mohr-Coulomb yield criteria and global buckling conditions were 
included in the models in order to predict collapse. Suiker (2018) introduced a socalled mechanistic model for straight walls that included a plastic yield condition and elastic buckling to describe the collapse modes. Finally, Kruger et al. (2019) proposed an analytical lower bound model based on a Mohr-Coulomb material with rheological material parameters as input.

Common for all of the mentioned 3DCP references is that they report good agreement between their suggested models and actual 3DCP tests. However, there is still room for improvement in the modelling.

In the present paper a novel numerical model to describe the early age behavior of 3DCP is suggested. The 3D print is modelled in the commercial software ABAQUS including a constitutive model describing a viscoelastic material on incremental form. The model input parameters are initially determined from conventional concrete tests and then subsequently adjusted through an optimization loop minimizing the difference in the displacements predicted by the ABAQUS model and displacement measurements performed by Digital image correlation (DIC) on the 3D print.

The novelty of the present work consist in the optimization of the input parameters by use of DIC measurements and the use of an incremental viscoelastic model. The suggested model is, however, currently in its implementation phase. Thus, the current paper is written to present the ideas of the suggested model and to illustrate its principles by use of a simple (current state) model.

\section{$2 \quad$ Numerical Model Definition}

The suggested model is comprised of the following elements: i) A numerical model in the commercial FEM program ABAQUS including kinematic nonlinear effects, ii) an incremental constitutive equation iii) and an optimization loop used to fit the input parameters. In the following a descriptions of the applied constitutive model and the optimization loop are given.

\subsection{Incremental Constitutive Equation}

The constitutive equations are considered on incremental form. The general form is given as, see e.g. Bazant et al. (1997);

$$
\underline{\dot{\sigma}}=\underline{D}_{t}\left(\underline{\dot{\varepsilon}}-\underline{\dot{\varepsilon}}_{k}\right)
$$

Where $\underline{\dot{\sigma}}$ represents the stress increment vector, $\underline{\underline{D}}_{t}$ is the constitutive material tangent stiffness matrix, $\underline{\dot{\varepsilon}}$ is the total incremental strain vector and $\dot{\varepsilon}_{k}$ is the incremental strain vector from e.g. creep, shrinkage and thermal gradients.

In the present study the 3DCP material is considered a viscoelastic material represented by a rheologic model. In the given case a generalized Maxwell material with $\mathrm{N}$ branches (each containing a springs and a dashpot in series) is considered, see Fig. 1. 


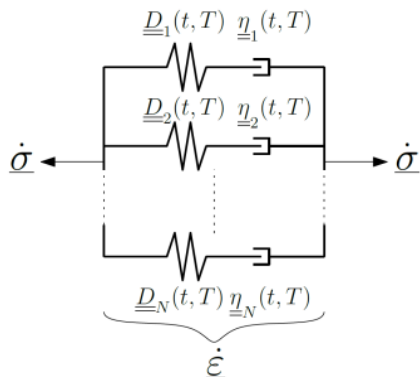

Figure. 1. Maxwell material dependent on time, $t$, and temperature, $\mathrm{T}$.

$\underline{\underline{D}}_{i}(t, T)$ and $\underline{\underline{\eta}}_{i}(t, T)$ represent a stiffness matrix and a viscosity matrix of the i'th branch, respectively. All parameters are functions of time, $t$, and temperature, $\mathrm{T}$.

Following the principles of the work in Bazant et al. (1997) it is possible to arrange a differential equation governing the total strain increments for each of the $\mathrm{N}$ generalized Maxwell branches. These are;

$$
\underline{\dot{\varepsilon}}=\underline{D}_{i}^{-1}\left[\left(\underline{\underline{\eta}}_{i}+\underline{D}_{i}\right) \underline{\dot{\varepsilon}}_{i}^{d}+\underline{\eta}_{i} \ddot{\ddot{\varepsilon}}_{i}^{d}\right], \quad i \in[1, N]
$$

where $\dot{\varepsilon}_{i}^{d}$ and $\ddot{\varepsilon}_{i}^{d}$ represent the i'th velocity and acceleration strain vector belonging to the i'th dashpot. The differential equations in Eq. (2) are solved numerically by discretizing time into $\mathrm{M}+1$ discrete points each separated by a time step $\Delta t_{n \rightarrow i+1}=t_{n+1}-t_{n}, n \in[0, M]$.

It is then assumed that the material stiffness and the damping matrices are constant in each time step. One choice could be to select the values in the middle of a time step as the constant values;

$$
\underline{\underline{D}}_{i}=\underline{D}_{i}\left(t_{j+1 / 2}, T_{j+1 / 2}\right), \quad \underline{\tilde{\eta}}_{i}=\underline{\underline{\eta}}_{i}\left(t_{j \rightarrow j+1 / 2}, T_{j+1 / 2}\right), \quad \underline{\dot{\eta}}_{i}=\underline{\underline{\eta}}_{i}\left(t_{j \rightarrow j+1 / 2}, T_{j+1 / 2}\right)
$$

Furthermore, the strain velocity and the strain acceleration present in Eq. (2) are assumed to, respectively, vary linearly in a time step and to be constant in time. This is formulated as

$$
\begin{gathered}
\dot{\varepsilon}_{i}^{d}(t)=\dot{\varepsilon}_{i, j}^{d}+\ddot{\varepsilon}_{i, j \rightarrow j+1}^{d}\left(t-t_{j}\right), \quad t_{j} \leq t \leq t_{j+1} \\
\ddot{\varepsilon}_{i}^{d}(t)=\ddot{\varepsilon}_{i, j \rightarrow j+1}^{d}, \quad t_{j} \leq t \leq t_{j+1}
\end{gathered}
$$

By integrating Eqs. (2), (4) and (5), the stress increment in each Maxwell branch in a time step is expressed as

$$
\Delta \underline{\sigma}_{i, j \rightarrow j+1}=\underline{\tilde{\dot{\eta}}}\left(\Delta t_{j \rightarrow j+1} \dot{\dot{\varepsilon}}_{i, j}^{d}+1 / 2 \Delta t_{j \rightarrow j+1}^{2} \ddot{\varepsilon}_{i, j \rightarrow j+1}^{d}\right)+\underline{\underline{\dot{\eta}}} \Delta t_{j \rightarrow j+1} \ddot{\varepsilon}_{i, j \rightarrow j+1}^{d}
$$


where the constant strain acceleration vector $\ddot{\varepsilon}_{i, j \rightarrow j+1}^{d}$ is given by the expression;

$$
\underline{\ddot{\varepsilon}}_{i, j \rightarrow j+1}^{d}=\left[\left(\underline{\underline{\dot{\eta}}}_{i}+\underline{\underline{D}}_{i}\right) \Delta t_{j \rightarrow j+1}^{2} / 2+\underline{\tilde{\eta}}_{i} \Delta t_{j \rightarrow j+1}\right]^{-1}\left(\underline{\underline{D}}_{i} \Delta \underline{\varepsilon}-\Delta t \underline{\dot{\varepsilon}}_{i, j}^{d}\left(\underline{\underline{\dot{\eta}}}+\underline{\tilde{D}}_{i}\right)\right)
$$

The total stress increment in the Maxwell material is found by adding all stress contributions from all branches together.

$$
\Delta \underline{\sigma}_{j \rightarrow j+1}=\sum_{i=1}^{N} \Delta \underline{\sigma}_{i, j \rightarrow j+1}
$$

The strain increment vector $\Delta \underline{\varepsilon}$ is the unknown parameter iteratively changed until equilibrium is reached. This iteration process is controlled by ABAQUS when implementing the constitutive relation as a sub function into the program. The material parameters in $\underline{\underline{D}}_{i}, \underline{\tilde{\eta}}$ and $\underline{\tilde{\dot{\eta}}}$ are input parameters provided by the user.

\subsection{Optimization Loop for Input Parameters}

In Fig. 2 an optimization loop suggested to improve the input parameters in the ABAQUS model is visualized. It consists of an optimization algorithm, an ABAQUS simulation and DIC displacement data from a 3D print.

The purpose of the optimization loop is to minimize the difference between the DIC displacements and the ABAQUS displacements by changing the input parameters. The initial guesses for the stiffness and viscous parameters are evaluated from conventional concrete tests.

The input parameters are inserted into the ABAQUS model, which then simulates the $3 \mathrm{D}$ print response. Next, the displacements from the ABAQUS model and the DIC measurements from the actual 3D print are compared. The comparison represents the object function, which the optimization algorithm aims to minimize by adjusting the input parameters. The iteration loop ends when the object function is minimized.

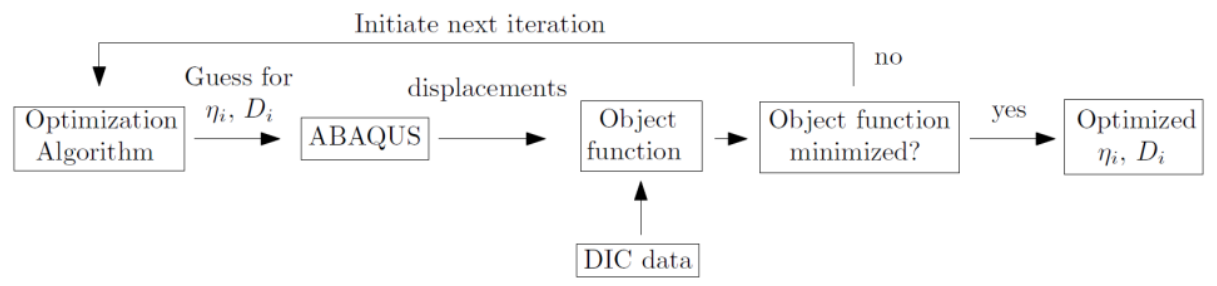

Fig. 2. Optimization loop.

\subsection{Present Model Status}

Currently the model suggested in the previous subsections is not fully implemented. In the current state the stiffness and damping parameters are constant with respect to time, $t$, and temperature, T. Furthermore, no optimization algorithm is added to the 
optimization loop. Thus, the results presented in the following sections are based on a simplified (current state) version of the suggested model.

\section{$3 \quad$ Materials and methods}

This section describes a 3DCP, including DIC measurements, performed at Danish Technological Institute used to exemplify the precision of the suggested numerical model.

\subsection{Materials}

The mortar used in the 3DCP experiments includes CEM I 52,5 R - SR 5 (EA) white cement (Aalborg Portland), Calcium Aluminate Cement (Ternal White ${ }^{\text {) }}$ ), limestone filler, fine sand (maximum particle size $0.5 \mathrm{~mm}$ ), water, and admixtures (a viscosity modifying agent, a high-range water-reducing agent and a hydration retarder). The mortar was prepared with an Eirich Intensive Mixer Type R08W.

Table 1. Mixing procedure for the base OPC-system.

\begin{tabular}{lc}
\hline Steps & Mixing time [s] \\
\hline Mix dry sand and 30\% water & 60 \\
Pause mixer and add the cement and limestone filler & - \\
Mix all material and add 60\% water & 120 \\
Pause mixer and scoop material attached to the blade and pan walls. & - \\
Add the admixtures and 10\% water & - \\
Continue mixing & 240 \\
\hline
\end{tabular}

The mix design has a water-to-binder ratio of $0.39,0.10 \%$ of plasticizer and $0.10 \%$ of viscosity modifying agent bwoc. Such OPC-system is then combined to a Calcium Aluminate slurry (at a binder to water ratio set at $1: 0.59$ ) to enable sufficient structural build-up necessary to stack layers. The mixing protocol (see Table 1) comprises a 7.0 min mixing for the base OPC-system, followed by a $1.5 \mathrm{~min}$ mixing for the Calcium Aluminate slurry. Note that i) a resting period of $15 \mathrm{~min}$ is included before the slurry is added and b) the slurry is mixed in batches prior to adding the mortar to the pump.

\subsection{D Printing Experiments}

The 3DCP experiments were carried out at the High-Tech Concrete Lab at the Danish Technological Institute. The 3D printing apparatus comprises a 6-axis industrial robot (FANUC R-2000iC/165F) and a progressive cavity pump (NETZSCH). The fresh mortar initially placed in a hopper is pumped into a rubber hose $(3.0 \mathrm{~m} \mathrm{long}, \varnothing 32$ $\mathrm{mm}$ ) that is attached to a custom designed extrusion nozzle with $\varnothing 20 \mathrm{~mm}$. 
The experiments consisted in printing prismatic elements with a fixed geometry suggested in Suiker (2018), see Fig. 3. The print collapsed after adding layer 24.

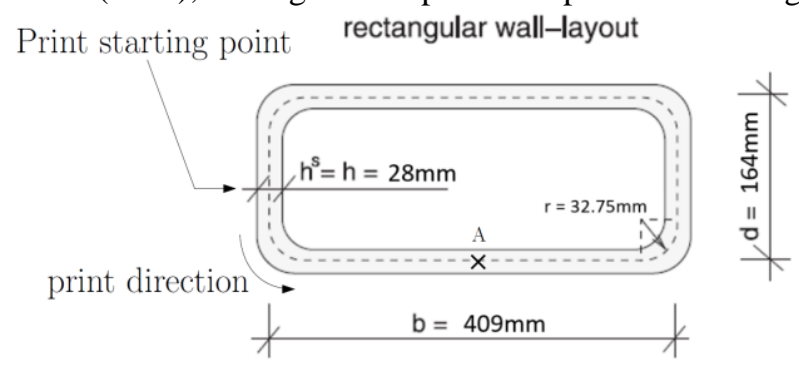

Figure. 3. 3D print geometry, from Suiker (2018).

Point A in Fig. 3 marks the point in which the DIC measurements for the optimization of the numerical input parameters are selected. The starting point of the print was in the middle of a side of length $\mathrm{d}$, and with a counter clockwise printing direction. The important printing process parameters are listed in Table 2.

Table 2. Processing parameters of the printing experiments.

\begin{tabular}{lc}
\hline Process parameters & Value \\
\hline Nozzle diameter, $D[\mathrm{~mm}]$ & 20.0 \\
Nozzle height, $H[\mathrm{~mm}]$ & 10.0 \\
Printing speed, $V[\mathrm{~mm} / \mathrm{s}]$ & 60.0 \\
Vertical build-up rate, $V_{H}[\mathrm{~m} / \mathrm{h}]$ & 2.00 \\
Extrusion flow rate, $U\left[\mathrm{dm}^{3} / \mathrm{min}\right]$ & 0.89 \\
Material density, $\rho\left[\mathrm{kg} / \mathrm{m}^{3}\right]$ & 20742 \\
Time per layer, $\mathrm{T}_{\mathrm{L}}[\mathrm{s}]$ & 18 \\
\hline
\end{tabular}

\subsection{Digital Image Correlation}

Digital image correlation (DIC) measurements were used to measure surface displacements in a particular region of interest in the printed specimens throughout the printing process.

Two digital single-lens Canon EOS 5DS cameras (50.6 megapixel) with a macro lens $(24$ or $100 \mathrm{~mm}$ ) were used to capture images of a surface of the continuously printed mortar structure. A surface picture was taken every 5 seconds at the same time with both cameras. Camera 1 had a $100 \mathrm{~mm}$ lens and the focus area of this camera was the first 12 - 14 layers of the printed structures (see Fig 4a). The pictures started simultaneously with the printing and continued to record throughout the whole printing process. Camera 2 had a $24 \mathrm{~mm}$ lens and the focus area of this camera was bigger, more precisely - all front surface of the printed structure (see Fig. 4a). As Camera 1 was focused on a smaller area, more detailed measurements were expected to be possible to extract from these pictures. Additional lights were provided to the test setup 
making sure that a high quality images could be captured of the printed surface, see Fig. 4b.

Afterwards, the images were post-processed using commercially available DIC software GOM Correlate. The post processing allowed to measure the full field surface displacements of the focused area that occurred while the printing of the structure was continued.
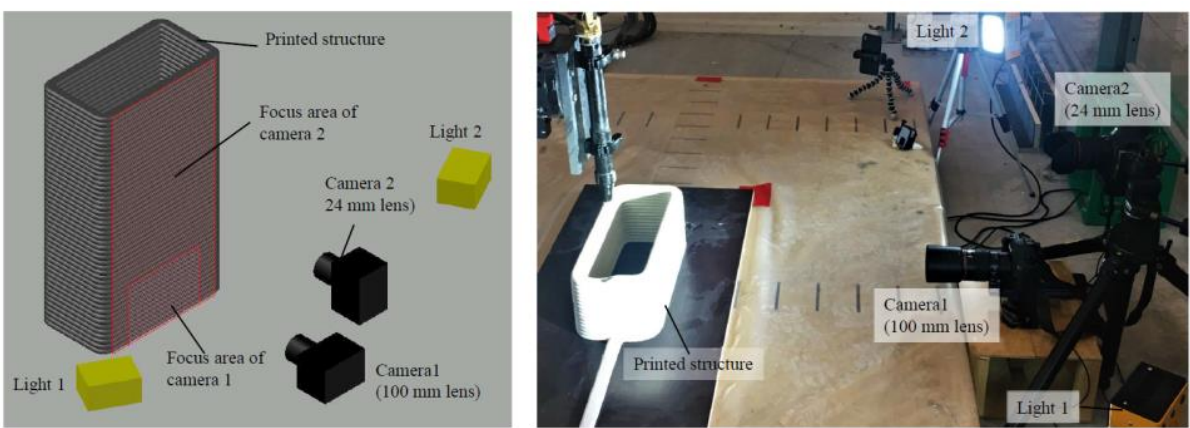

Figure 4 Test setup for DIC measurements: (a) sketch of the setup with highlighted measurement (focus) areas for each camera; (b) photo of actual setup.

\section{$4 \quad$ Numerical Model Validation}

The suggested numerical model used to predict the response of the 3D prints is presented. Furthermore, the results of the model predictions are presented and discussed.

\subsection{ABAQUS Model}

In order to minimize the computational time only the straight wall of length $b$ in Fig. 3 is modelled in ABAQUS. The ends of the wall are restricted against movement in the printing direction (x-direction), see Fig. 5. Furthermore, the bottom of the first print layer is restricted against translations in all directions. A six-noded wedge element with linear interpolation and full integration, denoted C3D6, is used for the meshing.

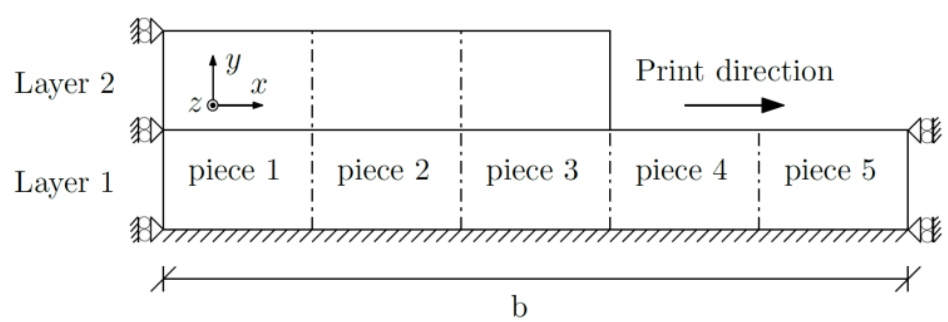

Figure 5 Sketch of boundary conditions and print 'pieces' in 3D print simulations. 
The model divides the wall in five 'pieces' of equal length, see Fig 5. Each 'piece' is added one at a time separated by a time interval corresponding to printing the piece. The applied constitutive model contains a single Maxwell branch, i.e. one spring stiffness and one dashpot in series. Both of the values are constant in the given case.

\subsection{Optimization of Input Parameters}

For the optimization process, the distance increment $\Delta \mathrm{u}\left(\mathrm{t}-\mathrm{t}_{0}\right)$ between the center of layer 1 and layer 2 in point A (cf. Fig. 3) is considered. This is sketched in Fig. 6, where $t_{0}$ refers to the point in time where the comparison between the DIC measurements and ABAQUS simulation results is started. In the given case the comparison is started when the print of layer four is initiated, i.e. $t_{0}=3 \mathrm{~T}_{\mathrm{L}}$. As stated previously, the optimization loop has not been implemented yet. Thus, the fitting of the input parameters is done manually.

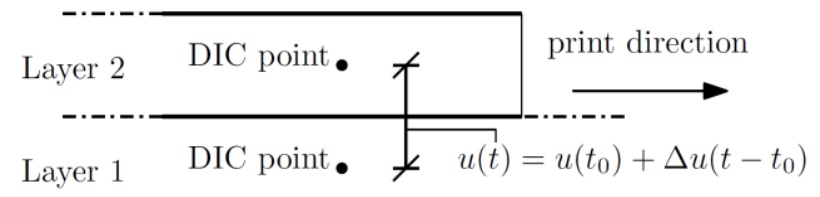

Figure 6 Distance between the center points of layer 1 and layer 2.

In Table 3 the parameters found from the fit are listed. It was selected to fit the parameters to the DIC measurements in the interval where layer four to six were printed. The initial value of the $\mathrm{D}_{1}$ was taken as $1 \mathrm{e} 5 \mathrm{~Pa}$, which corresponds to the order of magnitude measured from a rheometry test of the print material following the principles in Leal da Silva (2020).

Table 3. Fitted input parameters for ABAQUS model.

\begin{tabular}{cc}
\hline Input parameters & Value \\
\hline $\mathrm{D}_{1}[\mathrm{~Pa}]$ & $3 \mathrm{e} 4$ \\
$\eta_{1}[\mathrm{~Pa} \cdot \mathrm{s}]$ & $1 \mathrm{e} 8$ \\
\hline
\end{tabular}

In Fig. 7 the distance increments from the DIC measurements and the ABAQUS simulations including the parameters in Table 3 are shown. The vertical axis represents displacements and the horizontal axis represent time divided by $\mathrm{T}_{\mathrm{L}}$ (which corresponds to number of printed layers). The timeline is started when printing of the fourth layer is initiated.

From Fig. 7 it is seen that the DIC displacements and the ABAQUS displacements are in the same order of magnitude. A significant decrease in $\Delta \mathrm{u}\left(\mathrm{t}-\mathrm{t}_{0}\right)$ occurs in the DIC measurements during the printing of the fourth layer. However, when the following layers are added, only minor changes are seen. Thus, the material stiffness seems to increase significantly after compaction. 


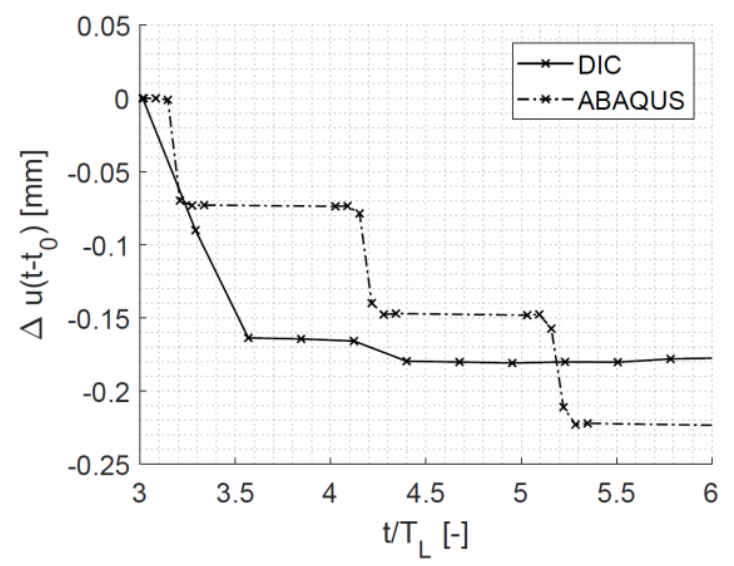

Figure 7 Distance increment $\Delta \mathrm{u}\left(\mathrm{t}-\mathrm{t}_{0}\right)$ based on DIC measurements and ABAQUS simulations, with $\mathrm{t}_{0}=3 \mathrm{~T}_{\mathrm{L}}$

The ABAQUS displacements, on the other hand, are seen decrease by the same order of magnitude every time a new layer is added directly on top of point A considered for the optimization $\left(\mathrm{t} / \mathrm{T}_{\mathrm{L}} \approx 3.25,4.25,5.25\right)$. This is expected, since the constitutive model contains a constant spring parameter, which does not take curing of the material into account. The most significant decreases in the DIC measurements takes place around $t / T_{L} \approx 3.25,3.5$, i.e. immediately when loading is added directly on top of point $\mathrm{A}$ and shortly after.

\subsection{Model Verification and Discussion}

As previously reported, the 3D print collapsed in the wall part of length $\mathrm{b}$ after stacking 24 layers, see Fig. 8. By use of the optimized parameters in Table 3 ABAQUS predicted a collapse at 10, layers. This corresponds to a deviation of $58 \%$ compared to the actual 3D print observations.

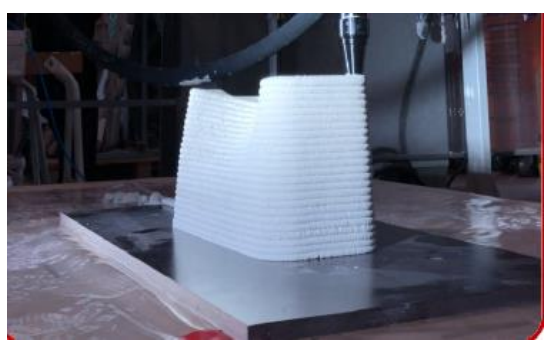

Figure 8 Collapse of 3D print at Danish Technological Institute (24 layers).

The significant deviation was, however, expected, since the numerical model applied is very simple. First of all only one set of (constant) input parameters are used. Furthermore, they only fitted to match the interval where layer four to six are printed 
(cf. Fig. 7). A significant improvement in the ABAQUS modelling of the 3D print is expected when the input parameters become a function of time and temperature. This will e.g. allow the model to include aging effects in the material.

\section{Conclusion}

An improved numerical model to simulate 3DCP was suggested. It consisted of an ABAQUS model including an incremental constitutive equation describing a viscous material and an optimization loop used to improve the model input parameters by use of DIC measurements. Currently the suggested model is only partly implemented. A simplified model was, therefore, used to illustrate the principles of the suggested model. An ABAQUS model including a single Maxwell branch with constant stiffness and damping parameters predicted the collapse of an actual 3D print at 10 layers. This corresponded to a relative error of $58 \%$ compared to the actual print observations whic was concluded to be a significant deviation, but also to be expected due to the simplified (current state) model used. Finally it was argued that future versions of the model are expected to improve the prediction of the 3D print behavior.

Acknowledgements. The authors would like to acknowledge the support of the Innovation Fund Denmark (Grant no. 8055-00030B: Next Generation of 3D-printed Concrete Structures)

\section{References}

1. Kruger, J., Zeranka, S., van Zijl, G.: 3D concrete printing: A lower bound model for buldability performance quantification. Automation in Construction 106 (2019): 102904.

2. Wolfs, R.J.M., Bos, F.P., Salet, T.A.M.: Triaxial compression testing on early age concrete for numerical analysis of 3D concrete printin, Cement and Concrete Composites (104), 103344 (2019).

3. Wolfs, R.J.M., Bos, F.P., Salet, T.A.M.: Early age mechanical behaviour of 3D printed concrete: Numerical modelling and experimental testing, Cement and Concrete Research (106), 103-116 (2018).

4. Suiker, A.S.J.: Mechanical performance of wall structures in 3D printing processes: Theory, design and experiements, International Journal of Mechanical Sciences (137), 145-170 (2018).

5. Buchanan, C. Gardner, L.: Metal 3D printing in construction: A review of methods, research, applications, opportunities and challenges. Engineering Structures (180), 332-348 (2019).

6. Bazant, Z.P., Hauggaard, A.B., Baweja, S., Ulm, F.-J., Microprestress-Solidification Theory for Concrete Aging and Drying Effects on Concrete Creep, Journal of Engineering Machanics, 11(123), 1188-1194 (1997).

7. Buswell, R., da Silva, W.R.L., Jones, S.Z., Dirrenberger, J.: 3D printing using concrete extrusion: A roadmap for research. Cement and Concrete Research, 112 (S.I.): 37-49, (2018).

8. Leal da Silva W.R., Fryda H., Bousseau JN., Andreani PA., Andersen T.J. (2020) Evaluation of Early-Age Concrete Structural Build-Up for 3D Concrete Printing by Oscillatory 
Rheometry. In: Di Nicolantonio M., Rossi E., Alexander T. (eds) Advances in Additive Manufacturing, Modeling Systems and 3D Prototyping. AHFE 2019. Advances in Intelligent Systems and Computing, vol 975. Springer, Cham 